\title{
ACUMULACIÓN POR DESPOSESIÓN Y RESPUESTAS LOCALES EN EL REMODELAJE DE LOS PAISAJES ESTUARIALES DEL SUR DE CHILE
}

\author{
ACCUMULATION BY DISPOSSESSION AND LOCAL RESPONSE IN THE \\ REMODELING OF THE ESTUARINE LANDSCAPES OF SOUTHERN CHILE
}

\author{
Debbie E. Guerra M. ${ }^{1}$ y Juan Carlos Skewes V. ${ }^{2}$
}

\begin{abstract}
En tres estuarios de la Cordillera de la Costa del sur de Chile se han verificado expresiones de resistencia frente a la expansión capitalista, materializadas en configuraciones paisajísticas diversas. El medio provee insumos a las poblaciones locales para poner coto y sortear dicho avance fundado en la desposesión. La comparación de tres escenarios costeros (ríos Lingue, Chaihuín y Llesquehue) evidencia que: (i) La acumulación por desposesión es multiforme, (ii) Los recursos históricamente establecidos como parte del paisaje condicionan el curso de tales procesos y (iii) La acumulación se expresa paisajísticamente de modo diferencial, condicionando el tipo de entramado social de las poblaciones locales, su futuro y su sustentabilidad. A partir del análisis de estas dimensiones se formula una tipología que permite caracterizar las respuestas locales ante la expansión capitalista.

Palabras claves: ecología política, acumulación por desposesión, expansión capitalista en Chile, estuarios y paisajes costeros.

Expressions of an intense resistance to capitalist expansion have been verified in three estuaries in Chilean's Southern Coastal Range, expressions that are materialized in diverse landscape configurations. The environment furnishes residents with resources that help them to stop and to limit the expansion based on dispossession. The comparison of three estuarine environments (rivers Lingue, Chaihuín and Llesquehue) makes evident that: (i) Accumulation by dispossession is multifarious, (ii) The resources established historically as part of the landscape shape the outcome of such processes, and (iii) Accumulation finds diverse forms of expression in the landscape, conditioning the local population's social organization, sustainability and future. From this analysis we formulate a typology that allows us to characterize local responses to capitalist expansion.

Key words: Political ecology, accumulation by dispossession, capitalist expansion in Chile, estuaries and coastal landscapes.
\end{abstract}

Los estuarios de las regiones de Los Ríos y Los Lagos en el sur de Chile han sido transformados por la expansión capitalista y por la acción reguladora del Estado. Tales transformaciones ocurren en zonas que sirvieron de refugio a comunidades indígenas y suscitan acciones de resistencia de notoriedad pública, las que le permiten conservar cierta autonomía frente a las presiones externas. La expansión del mercado en estos territorios se basa en formas de acumulación por desposesión que, valiéndose de medios extraeconómicos, liberan recursos -incluyendo la fuerza de trabajo- para incorporarlos a la producción capitalista, privando a las poblaciones locales de la relación que históricamente han establecido con su medio (Adrienne 2008; Fine 2006; Harvey 2003; Little
1999; Sparke 2008). Esta acumulación “originaria es previa a la acumulación capitalista ('previous accumulation', como la llama Adam Smith), una acumulación que no es el resultado del modo de producción capitalista, sino su punto de partida" (Marx 1980: 891 [énfasis del autor]).

Las poblaciones locales se sirven de los recursos de los estuarios para poner coto y, en parte, sortear la expansión capitalista. La comparación de los ríos Lingue, Chaihuín y Llesquehue sugiere que la acumulación por desposesión es multiforme y que la resistencia local condiciona el curso de tal expansión, estableciendo las bases para la sustentabilidad de las poblaciones estuariales, con lo que es posible identificar los fundamentos para la reposesión de territorios hoy amenazados.

\footnotetext{
1 Instituto de Enfermería Materna, Universidad Austral de Chile, Valdivia, Chile. Carelmapu 2225, Valdivia. Código Postal 5090984.dguerra@uach.cl

2 Instituto de Ciencias Sociales, Universidad Austral de Chile, Valdivia, Chile. jskewes@uach.cl
} 


\section{Acumulación por Desposesión}

David Harvey (2006: 153) entiende la acumulación por desposesión como una prolongación de las prácticas descritas por Marx en los orígenes del capitalismo e incluyen la privatización de la tierra; la expulsión de poblaciones campesinas; la conversión de las distintas formas de propiedad en propiedad privada; la supresión de los recursos comunales; la eliminación de formas alternativas de producción y consumo; la apropiación colonial de los recursos naturales; la monetarización y la tributación; el tráfico de seres humanos; la usura y el endeudamiento a través del crédito. El Estado, con su monopolio de la violencia y de la definición de la legalidad, juega un papel crucial en promover estos procesos que terminan con la disociación entre el productor y sus medios de producción y con la sustitución de las estructuras sociales preexistentes por relaciones capitalistas de producción.

Las condiciones sociales para la emergencia de este régimen requieren de connivencia con actores locales (Marx 1980) y las dinámicas de poder (incluyendo las relaciones étnicas y de género) facilitan la desposesión por la vía de la opresión extraeconómica y la instalación de un sistema capitalista propiamente tal (Sparke 2008).

El enredo es una estrategia de usurpación cuyo papel se torna crítico en escenarios donde las formas asociativas tradicionales limitan la incursión de intereses extralocales ${ }^{1}$. La liberación de recursos exige la separación de los medios de producción y de las relaciones sociales que hacen posible formas de producción precapitalistas (Turner 2008). La ruptura es doble: del medio y de los semejantes (Harvey 2006; Taussig 1980). Así como lo sugiere Malinowski (1966:157 [traducción de los autores]): "Al remover a un ser humano de su ambiente social, ipso facto se le depriva de todo su soporte moral, eficiencia económica e incluso interés por la vida".

El olvido pasa a ser un factor decisivo: " ¿Acaso no es olvido el progresivo extrañamiento de las personas en relación a las cosas que ellas producen y a quienes les rodean?" (Eiss 2008: 81 [traducción de los autores]). La naturalidad con que el capitalismo se presenta resulta del desvanecimiento de la conciencia que percibía el orden social como un tipo particular de artificio, uno con una historia humana (Eiss 2008). Las relaciones sociales ensamblan los sistemas que organizan la vida cotidiana, creándose sujetos funcionales a tales fines (Turner 2008).
La acción del Estado es decisiva en la reorganización de los territorios que se ponen a disposición del desarrollo capitalista, donde el fraccionamiento de la población es un requisito indispensable. Tal acción provoca la resistencia de la clase subordinada, sea para mitigar las exigencias hechas por las clases dominantes o para avanzar en sus propios intereses (Scott 1985: 290), resistencia que es especialmente significativa en la arena local (Skewes y Guerra 2004).

La acumulación por desposesión se analiza en tres paisajes estuariales: (i) El uso del mar para desechos industriales de una planta de celulosa con impacto directo sobre las poblaciones aledañas al río Lingue; (ii) La regulación en el acceso a los recursos bentónicos y su efecto en las poblaciones del estuario Llesquehue, y (iii) Las transformaciones producidas por la actividad forestal en las zonas aledañas al río Chaihuín.

En el pasado la desposesión se dio aquí por despojo directo, corridas de cerco y engaño ante los tribunales (ver Bengoa 1996; Chihuailaf 1999; Molina et al. 2006; Vergara, et al. 1996). Hoy se agregan la desposesión por contaminación (depleción de los recursos naturales por la acción de contaminantes asociados a actividades industriales); por regulación (limitación en el acceso a los recursos naturales por disposiciones legales como la Ley General de Pesca y Acuicultura No 18.892 de 1991 y sus modificaciones), y por ordenamiento territorial (reubicación de las poblaciones y actividades económicas en los territorios de acuerdo a criterios de racionalidad productiva), modalidades que se asocian a los sectores más dinámicos de la economía global: la celulosa, la madera y la pesca (Ridgeway 2004), y son sancionadas y validadas legalmente a través de estudios de impacto ambiental (según lo establece la Ley de Bases del Medio Ambiente $\mathrm{N}^{\circ} 19.300$ de 1994), la delimitación de áreas de manejo de recursos bentónicos e instrumentos de planificación territorial.

\section{La Construcción del Paisaje}

La acción humana es parte del paisaje y encuentra expresión en él a través de las modificaciones, creaciones y percepciones del entorno, incluyendo las dimensiones materiales, inmateriales, discursivas y emocionales que intervienen en su modelado (Crumley 1994b; Sauer 1925; Sellin 1996; Stokowski 2002). El paisaje es fuente de identidad, permite 
mantener distinciones y lazos sociogeográficos y manifiesta la fecundación recíproca entre el pasado y el presente, permitiendo estudiar la estructura, funcionamiento y cambios en áreas heterogéneas (Crumley 1994b; Lipietz 2002; Stokowski 2002; Thornton 1997). Las prácticas sociales y las construcciones ideológicas son constitutivas del paisaje, como lo es la interrelación entre grupos con distintas orientaciones culturales y productivas (Balée 1998; Crumley 1994a; Descola 1996; Lipietz 2002; Patterson 1994).

El estudio etnográfico e histórico de los complejos encadenamientos de causalidad mutua en la relación entre humanos y medio ambiente permite abordar el carácter desigual que distintas concepciones del entorno desempeñan en su modelado (Balée 1998; Crumley 1994a; Escobar 1999; Lipietz 2002). El paisaje entraña contradicciones entre grupos con estrategias adaptativas o proyectos específicos que les llevan a competir por el acceso a los recursos y por el dominio territorial (McGovern 1994). El paisaje definido como un "producto sociocultural creado por la objetivación sobre el medio y, en términos espaciales, de la acción social tanto de carácter material como imaginario" (Criado 1999:9) sintetiza estas contradicciones.

La configuración paisajística resulta de prácticas de dominación y resistencia, donde las segundas viabilizan la mantención de espacios multifuncionales (Klein y Wolf 2007). Esta multifuncionalidad opera de modo heteróclito y vincula contingentemente sea por espacialidad, temporalidad o imbricación económica, social o cultural, a los distintos actores (Vasey 1998).

El paisaje es la síntesis simbiótica entre seres humanos y naturaleza (Fischer-Kowalski 2002), $\mathrm{y}$, a diferencia de la sociedad o del ambiente, entraña la asociación evanescente de significados e hitos, importando la lugarización del mundo (Tuan 1977). Tiene, pues, un carácter relacional que establece vínculos entre hitos y personas en las dimensiones ecológicas, sociales y simbólicas (Hirsch y O’Hanlon 1995; Zedeno et al. 1997). El estudio del paisaje considera tanto las dimensiones naturales como estéticas, morales y psicológicas, la omisión de alguna lleva a perder el punto de vista fundamental para entenderlo: el sentido que tiene para el ser humano que lo habita (Aasbŏ 1999). La oposición entre el mundo de vida y la perspectiva globocéntrica cobran aquí su valor interpretativo (Ingold 1995).
Los paisajes locales enfrentan procesos globales que operan sobre la base del desenraizamiento de las relaciones sociales (Eiss 2008; Polanyi 1944; Turner 2008). El paisaje se pliega ante presiones provenientes de los mercados mundiales y frente a la acción de gobiernos que procuran facilitar su implementación, pero también ante la impronta de quienes no participan de tales intereses y que, por el contrario, intentan persistir en sus mundos de vida, forjar identidades, conservar patrimonios o generar alternativas propias. Los conceptos de jerarquía y de hetarquía dan cuenta de las dinámicas locales desde una perspectiva ecológica y su interacción permite comprender de mejor forma el dinamismo del paisaje. Las heterarquías son sistemas complejos en que los elementos tienen "el potencial de no estar ordenados en relación a otros elementos u ordenados de manera variable dependiendo de los requerimientos del sistema" (Crumley 1994a:12 [traducción de los autores]). En contraste, los sistemas jerárquicos son aquellos en los que algunos elementos del paisaje se subordinan a otros de un modo centralizado. Los procesos de centralización son propios de actividades que establecen eslabonamientos productivos fundados en la apropiación de insumos generados por otras unidades a las que someten y por las inversiones que trocan en recurso a la naturaleza, en oposición a las prácticas de autoconsumo características de economías campesinas (Gudeman 2008).

Las formas y contornos paisajísticos tienen fronteras porosas que son infiltradas por el flujo globalizante, pero su elasticidad permite la persistencia en territorios que, de lo contrario, se mercantilizarían a cabalidad. Los paisajes se deben analizar, pues, sobre la base de las fuerzas globales, las conexiones locales y las culturas que les dan sentido (Zsuzsa y Ó Riain 2002).

\section{Enjambres Locales}

Los paisajes estuariales de las vertientes occidentales de la Cordillera de la Costa, entre los ríos Lingue y Contaco son espacios que albergaron a poblaciones expulsadas de los llanos centrales. $\mathrm{La}$ zona corresponde a un ecotono entre los ecosistemas marino y boscoso y su altura disminuye de norte a sur, dando lugar a una serie de aterrazamientos en los sectores costeros (Subiabre y Rojas 1994:33). El clima es templado lluvioso, con precipitaciones anuales que superan los $1.800 \mathrm{~mm}$, y con una 
temperatura que oscila entre los $10 \mathrm{y} \operatorname{los} 12^{\circ} \mathrm{C}$. La franja costera es una zona compacta y las bahías se encuentran separadas por roqueríos de difícil acceso, limitando el poblamiento humano a las zonas de desembocaduras de ríos y estuarios (Duhart et al. 2001). Sus cursos de agua -sometidos al régimen mareal- se caracterizan por su productividad biológica (Perillo 1995; Short 1979) (Figura 1).

Predominan los suelos rojo-arcillosos, altamente erosivos, de uso forestal (Subiabre y Rojas 1994). El bosque templado lluvioso se distribuye en asociaciones boscosas siempreverdes incluyendo bosques de olivillo (Aextoxicon punctatum), de coihue-ulmo (Nothofagus dombeyi-Eucryphia cordifolia), y de tepa-tineo (Laurelia philipiana-Weinmannia dentata), que se han visto disminuidas por la agricultura, ganadería, la obtención de madera y los incendios forestales (Lara et al. 2003).

La actividad humana en estos territorios se asocia al bosque y a la recolección marina. Las especies de moluscos, explotadas hace 8.500 años a.p., son las mismas que se extraen en el presente (Moreno y Fedele 2003). Las poblaciones se atrincheran en pequeñas plataformas de la Cordillera de la Costa (Concha 1998; Foerster 2004; Vergara et al. 1996). Los regímenes de naturaleza resultantes (Escobar 1999) expresan la articulación de elementos caracterizados por su ductibilidad y resiliencia, no sometidos unos a otros ni ordenados en torno a un requerimiento centralizado.

Las poblaciones estuariales de los ríos Lingue, Chaihuín y Llesquehue, aun cuando no tienen vinculación directa entre sí, pertenecen a similar ecotono y encarnan trayectorias paralelas en términos de poblamiento: enfrentan procesos de transformación resultantes en divisiones internas a la par que surgen nuevos actores. Las coincidencias verificadas suponen ciertas regularidades en la ecología política de los estuarios. Estas poblaciones pertenecen a las comunas de Mariquina, Corral y San Juan de la Costa, la que es la más pobre y con mayor población indígena (59,3\%), ocupando el 2003 el último lugar en el Índice de Desarrollo Humano entre las 341 comunas del país (Ministerio de Planificación [MIDEPLAN 2003]). Corral es la comuna de menor tamaño (3.670 habitantes) y con el mejor índice de desarrollo humano: lugar 183 a nivel nacional. Mariquina es la más poblada (18.223 habitantes) y la que presenta mayor diferenciación interna en cuanto a actividades productivas (Tabla 1).

Tabla 1. Antecedentes generales de las comunas de Mariquina, Corral y San Juan de la Costa. General backgrounds of Mariquina, Corral and San Juan de la Costa counties.

\begin{tabular}{lccccc}
\hline & Ext. & Pob. & $\begin{array}{c}\text { Pob. } \\
\text { Mapuche }\end{array}$ & Lugar & $\begin{array}{c}\% \text { no } \\
\text { asistió }\end{array}$ \\
\hline Mariquina & 1.321 & 18.223 & $23,1 \%$ & 243 & 9,8 \\
Corral & 767 & 3.670 & $11,3 \%$ & 223 & 9,0 \\
$\begin{array}{l}\text { San Juan } \\
\text { de la Costa }\end{array}$ & 1.517 & 8.831 & $59,3 \%$ & 341 & 13,4 \\
\hline
\end{tabular}

Fuente: Elaboración propia a partir de la información proporcionada por el INE (2002) y MIDEPLAN (2003).

Las poblaciones de los estuarios Lingue (Maiquillahue, Mississippi, Mehuín Bajo), Chaihuín (Chaihuín, Cadillal, Huiro) y Llesquehue (Caleta Manzano, Choroy Traiguén y Pucatrihue) son poco numerosas, dispersas, con predominancia masculina e indígena y con bajas tasas de escolaridad (Tabla 2).

La metodología para acceder a cada uno de estos estuarios se basó en el contacto directo con las organizaciones locales entre los años 2006 y 2007. Se identificó a interlocutores claves, representativos de las distintas áreas de actividad económica y de la procedencia étnica, sexo y edad de la población local, a quienes se invitó a participar en grupos de discusión relativos a la situación actual de los estuarios (Tabla 3).

Tabla 2. Características demográficas de las poblaciones de los estuarios de los ríos Lingue, Chaihuín y Llesquehue. Demographic characteristics of the populations of Lingue, Chaihuín and Llesquehue rivers estuaries.

\begin{tabular}{lccccccc}
\hline \multicolumn{1}{c}{ Estuario } & Hombre & Mujer & Total & Pob. Mapuche & Ind. Masc. & Pob. $>60$ años & Escolaridad \\
\hline LINGUE & 366 & 303 & 669 & 59,9 & 120,8 & 15,7 & 5,1 \\
CHAIHUIN & 272 & 215 & 487 & 22,0 & 126,5 & 12,5 & 5,5 \\
LLESQUEHUE & 225 & 192 & 417 & 57,8 & 117,2 & 10,6 & 4,7 \\
\hline
\end{tabular}

Fuente: Elaboración propia a partir de la información proporcionada por el INE (2002). 


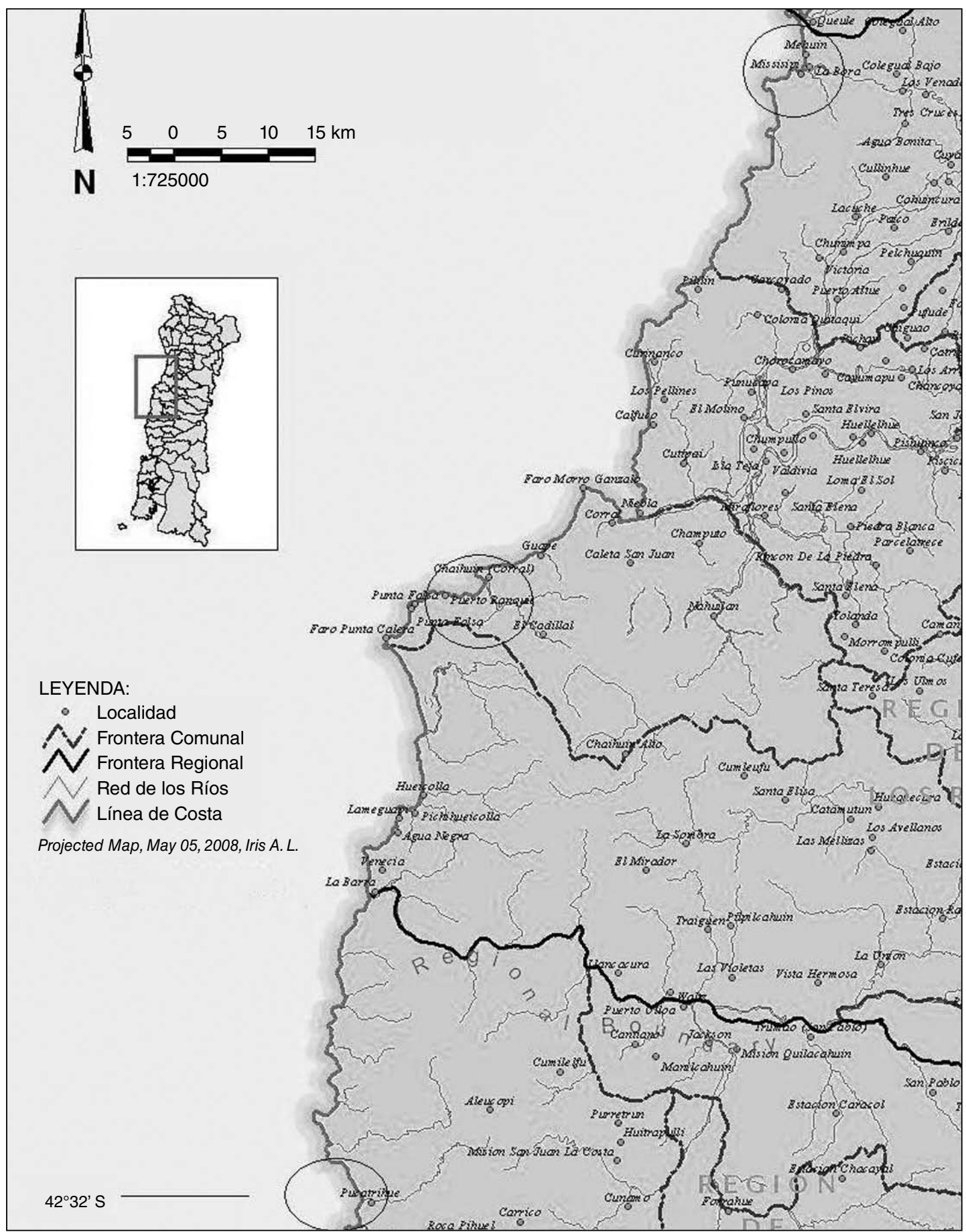

Figura 1. Las zonas costeras de las regiones de Los Ríos y Los Lagos. The coastal zones in the regions of the rivers and the lakes. 
Tabla 3. Distribución de la población entrevistada por estuario, sexo, generación y procedencia étnica.

Distribution of the interviewed population by estuary, sex, generation and ethnic origin.

\begin{tabular}{|c|c|c|c|c|c|c|c|c|}
\hline \multirow[b]{2}{*}{ Estuario } & \multicolumn{2}{|c|}{ Sexo } & \multicolumn{3}{|c|}{ Edad } & \multicolumn{3}{|c|}{ Etnicidad } \\
\hline & $\Sigma$ & L & $\begin{array}{l}\text { ల్ } \\
\stackrel{0}{0}\end{array}$ & $\frac{\stackrel{\rho}{3}}{\frac{7}{4}}$ & 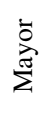 & $\frac{\widetilde{0}}{\stackrel{0}{\Xi}}$ & 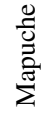 & $\begin{array}{l}\underset{\Xi}{\tilde{J}} \\
\stackrel{\Xi}{\mathbb{U}} \\
\end{array}$ \\
\hline Lingue & 2 & 3 & 2 & 2 & 1 & 1 & 4 & 0 \\
\hline Chaihuín & 3 & 2 & 1 & 3 & 1 & 2 & 3 & 0 \\
\hline Llesquehue & 3 & 3 & 2 & 2 & 2 & 1 & 4 & 1 \\
\hline Total & 8 & 8 & 5 & 7 & 4 & 4 & 11 & 1 \\
\hline
\end{tabular}

Fuente: Elaboración propia.

Estos estuarios tienen un carácter paradigmático pues entrañan: (i) La convergencia de actores diversos en territorios de confluencia ecológica e histórica, (ii) Notoriedad pública por la intensidad de los procesos allí vividos y (iii) Un desafío a las políticas públicas relativas tanto al medio ambiente como a las poblaciones indígenas.

\section{Acumulación por Desposesión, Nuevos Enredos y Consecuencias Locales}

\section{Desposesión por contaminación}

Por más de diez años, a partir de 1996, la población de la bahía de Maiquillahue frustró la instalación de un ducto para la evacuación de residuos de la Planta Valdivia de Celulosa Arauco. Diversos actores, tomando como plataforma simbólica el mar, se aglutinaron en torno a un discurso que integraba el conocimiento científico con el conocimiento práctico y la fe religiosa (Skewes y Guerra 2004). Sin embargo, al comenzar la segunda fase del conflicto en 2004, el paisaje local muta por la asignación de las áreas de manejo. Las áreas de manejo son espacios costeros destinados al manejo y explotación de recursos bentónicos, tanto de fondos duros como blandos, por parte de una organización de pescadores artesanales, legalmente constituida (Instituto de Fomento Pesquero [IFOP] 2009). El mar deja de ser una plataforma unitaria y la competencia por su control derivó en la formación de cuatro sindicatos, tres en Mehuín y uno en Mississippi.
Las áreas de manejo introducen un principio jerárquico: la primacía del sector pesquero en el control sobre el borde costero. Al reactivarse el proyecto del ducto en 2004, los pescadores asumen el liderazgo en la defensa del mar, marginando a sectores que fueron importantes en el decenio anterior (mujeres, comerciantes, jubilados). Su resistencia, no obstante, se desvanece cuando la empresa negocia con ellos su adhesión al proyecto, con lo que logra fragmentarlos. El 9 de octubre de 2007 se suscribe un convenio notarial declarando la necesidad que la empresa tiene para descargar sus residuos a través de un emisario submarino. Las partes se comprometen a colaborar en los estudios para la operación del ducto y en las gestiones necesarias para el uso de terrenos, playa y fondo de mar, declarando que dicho espacio no es necesario para el desarrollo de la pesca. La empresa compromete sobre quinientos mil dólares por la suscripción del Convenio, a razón de $\$ 3.000 .000$ por asociado, monto que se incrementa con el cumplimiento de las fases posteriores de construcción y operación del ducto y, eventualmente, cesan si el emisario se paraliza por algún evento externo, ajeno a la empresa.

La lógica del enredo rinde sus frutos, enmarañando las relaciones vecinales: el 20 de septiembre de 2007 se denuncia la amenaza de muerte contra dirigentes opositores a CELCO; a quienes aceptan el dinero de la empresa, son acusados de traidores. En adelante, serán más quienes firmen y la resistencia se desplaza hacia la caleta de Mississippi, donde se redefine el Comité de Defensa del Mar. Este desplazamiento es consistente con el régimen hetárquico que se vive al sur del río Lingue. La resistencia se concentra en un puñado de jóvenes y mujeres, guarecidos en una sede carente de protección, que vigilan el ingreso de embarcaciones. Es la voluntad para detener a la empresa en su obstinación por evacuar hacia el mar los desechos de su planta, desposeyendo a pescadores, buzos y algueros de su fuente de vida económica.

La posibilidad de solventar la resistencia mediante medios de autoprovisión da cierta autonomía a los grupos locales. El paisaje estuarial permite a las mujeres, cuya expresión de resistencia es la más radicalizada al momento del conflicto, combinar la recolección de orilla con la producción de sus huertas y el comercio, mientras los hombres se hacen cargo de las siembras, la leña, el buceo, el cultivo de 
choritos (Mytilus chilensis) y la pesca. Los jóvenes se integran a la resistencia en el período estival.

El cultivo de choros (Choromytilus chorus) y choritos (Mytilus chilensis) y la pesca de las demás especies de los estuarios sirve a quienes procuran escapar a las determinantes que les impone el mercado. "El río es fuente de vida", dice un entrevistado, "yo tengo un espacio chico donde saco choritos, ahí tengo un lugar que posiblemente hay que regularizarlo a mi nombre y también vamos a sacar un robalito (Centropomus nigrescens)". El choro sirve para autoabastecimiento, ahorro y moneda de cambio. Su cultivo es uno de los medios con que la comunidad resiste y al hacerlo recrea su relación con el paisaje estuarial que le sustenta.

La población se rearticula en torno a los cursos de agua: siete solicitudes de concesiones acuícolas se presentan el año 2007 frente a una sola en años anteriores, agregándose las peticiones de aprovechamiento de aguas que, desde 2004, se han incrementado en decenas. En Mehuín se suman 28 derechos de aprovechamiento de aguas concedidos y dos solicitudes de regularización en trámite en Mississippi. La Comunidad Indígena Piutril es titular de siete derechos de agua; las comunidades Coscoyen Rayen y Rayen Lafquen son titulares de uno cada una; la Comunidad Indígena Villa Nahuel de cinco y la Asociación Indígena Lonko Nahuel es titular de dos (Accatino 2009).

\section{Desposesión por división territorial}

Las dinámicas del poblamiento del río Chaihuín responden a la actividad forestal que, en la primera parte del siglo veinte, estuvo dedicada al abastecimiento de carbón y leña para la fundición acerera Altos Hornos de Corral (Skewes 2001). La presencia de la población indígena se reconoce en el Fundo Chaihuín, hacia el sur del río, la que es hostigada por los propietarios entre 1958 y 1988 (Vergara et al. 1996). Los antiguos vivientes perseveraron como una pequeña comunidad ganadera que complementaba su producción con la explotación del bosque y extracción de recursos del mar.

Al norte de la desembocadura del río Chaihuín, se ubican residentes y pescadores predominantemente chilenos; hacia el interior lo hacen pobladores chilenos (Cadillal Alto y Cadillal Bajo), quienes combinan la pequeña agricultura y ganadería con el trabajo de la leña y el cultivo de choritos (Mytilus chilensis). $\mathrm{Al}$ sur del río, en las inmediaciones del estuario, se ubica la comunidad de Huiro, predominantemente indígena, y cuya actividad principal es la crianza de animales combinada con la leña, la recolección de orilla y el buceo (Godoy 2003).

El cierre de los Altos Hornos, en 1958, creó condiciones para una relación horizontal entre quienes poblaban el territorio en su parte septentrional, al tanto que los habitantes de Huiro, al sur, ejercitaban una precaria autonomía. En 1988, parte del predio es adquirido por CAP Inversiones, con la intención de crear una planta de celulosa. Entre 1989 y 2000 se plantan 3.214,9 ha de eucaliptus (Eucaliptus globulus, E. nitens y E. delegatensis) (Tecklin y Farías 2003). La comunidad de Huiro recibe el apoyo del Obispado de Valdivia, creándose en 1994 la Fundación Manelowün Huiro. La empresa dona 730 ha con las que el Obispado procura regularizar la ocupación de los antiguos residentes. La donación favorece a 26 familias de Huiro y doce de Chaihuín.

En 2001 el Obispado suscribe un convenio con el Centro Forestal (CEFOR) para explotar el bosque de un modo sustentable. Se plantan 100 ha con especies exóticas y se propone la creación de una aldea para los comuneros. La iniciativa encuentra un fuerte rechazo por parte de los troncos familiares antiguos del territorio, quienes se resisten a la idea de ser radicados bajo una modalidad contraria a su tradición. El 6 de agosto de 2001, El Diario Austral de Valdivia señala que "la mayoría lo rechaza, puesto que los obligará a dejar sus actuales viviendas para trasladarse hacia otros sectores". Trece de catorce familias se retiran de la Comunidad de Huiro para formar su propia asociación (Comunidad Indígena Antillanca) (Godoy 2003; Le-Breton y Flores 2007).

La tierra se divide de acuerdo a criterios racionales, haciendo un "buen uso" del recurso y disponiendo a la comunidad de un modo consistente con tal uso. Al hacerlo queda en entredicho la lógica indígena que valora las formas colectivas de operar en el territorio. La desposesión se traduce en crecientes limitaciones en el acceso a la naturaleza y en competitividad entre actores, "algunas personas empezaron a sacar árboles, o leña, o madera, y sin pedir el parecer de los demás", dice un residente (Godoy 2003:25).

La división producida por la intervención externa agrieta las relaciones comunitarias. En 2004 se instaura la propiedad individual $\mathrm{y}$, no obstante, Huiro persiste en reafirmar su identidad en el vasto 
territorio dominado por el río Chaihuín. Ello se manifiesta en procesos de recuperación de tradiciones culturales y prácticas que les singularizan, y sobre la base de las que hacen un uso alternativo de los recursos locales, generan condiciones de mayor autonomía. A partir del año 2002 el Sindicato de Pescadores de Huiro obtiene la concesión de dos áreas de manejo y hoy son reconocidos como una organización exitosa en el manejo sustentable de los recursos del mar. Huiro cuenta con un centro etnoturístico único en la comuna de Corral, el cual sirve de asiento a la agrupación de mujeres artesanas Kutralhue, que integra a los sectores divididos de la comunidad, además de aprovechar los recursos locales produciendo mermeladas, hierbas para infusiones y un servicio de banquetería étnica. Este conjunto de iniciativas cuenta con el respaldo de organismos no gubernamentales como la World Wildlife Foundation, The Nature Conservancy y Agenda 21 y permite dar cuenta de procesos autonómicos que amortiguan el impacto de desarraigo que el ordenamiento territorial puesto al servicio de los intereses del mercado supone.

\section{Desposesión por regulación}

Las áreas de manejo de recursos bentónicos han regulado el acceso a los territorios costeros. Con ellas la autoridad ha procurado evitar la depredación producida por el explosivo desarrollo del mercado exportador de mariscos. La concesión de las áreas deja en manos de los sindicatos la explotación de los recursos bentónicos. Aunque esta medida ha frenado la devastación del loco (Concholepas concholepas), sus impactos sociales son una incógnita. La inversión pública favorece un polo de desarrollo que no se hace cargo de su entorno inmediato, el cual conserva su dinámica horizontal de funcionamiento.

La población indígena organizada en las comunidades Choroy-Traiguén y Purretrún-Pucatrihue se ubica en las inmediaciones del río Llesquehue y depende de la pequeña agricultura con crianza de animales, del trabajo de la leña y de la recolección de orilla. La abundancia de recursos marinos en las bocas de los estuarios ha estimulado prácticas productivas ancestrales a través del mareo. La práctica de mariscar desarrollada familiarmente y comandada por mujeres que avistando la luna y la baja de la marea salían a proveerse de las riquezas del mar ha sido de mucha importancia. La relación quedó míticamente establecida en la figura de Huenteao, protector de las comunidades huilliche, quien se casa con una mujer del mar, radicándose en la roca encantada de Pucatrihue (Foerster 1985; Quiroz y Olivares 1987; Skewes y Silva 2007).

La Ley General de Pesca sustituye esta relación creando las figuras de buzo, recolector de orilla y pescador artesanal y, al oficializar las prácticas, la presión por los recursos se proyecta en las relaciones locales. El Sindicato de Pescadores de Pucatrihue, creado en 1999 con 97 socios, goza de la exclusividad del área donde se emplaza la roca encantada del Abuelito. Del Sindicato sólo participan hombres y a la fecha cuenta con menos de la mitad de los socios originales. Desde 2001, tienen dos de las tres áreas de manejo de la zona, cuya cosecha complementa los ingresos familiares de los socios en una cifra cercana a los dos millones de pesos anuales (aproximadamente, 3.300 dólares). Con la formación del Sindicato, las mujeres e indígenas quedan excluidos de la actividad y comienzan conflictos entre pescadores por el robo de mariscos.

La privatización del mar desposee a los indígenas del acceso al lafkenmapu. Privatizar es privar a las comunidades indígenas de uno de sus recursos más ancestrales (Chihuailaf 1999). Los pescadores acusan a los indígenas de nunca haber practicado la pesca y aunque la ley les da la razón, desde el punto de vista de una práctica que por cientos de años ha vinculado a un pueblo con su mar, no la tienen. El Presidente del Sindicato plantea: "El Gobierno le dio la posibilidad a las comunidades indígenas que tienen mar de hacer trabajos de pesca". Y sigue: "Pero mucha gente de las comunidades que tienen mar, no tienen ningún pescador, ni buzos, ni nada" (entrevista del 29 de enero de 2007). La regulación separa la actividad pesquera del mundo a que pertenece. El negar al mapuche su condición de marero es amputar un ámbito de su quehacer productivo. El reconocimiento del uso consuetudinario de las costas a través de la elaboración de la Ley 20.249 constituye un oportunidad para enmendar (Lafkenche 2005). Empero, el cuerpo legal pareciera carecer de los medios administrativos para tornar realidad esta aspiración (Accatino 2009).

\section{Vinculaciones Globales y Consecuencias Locales}

La comparación de los casos permite evaluar el impacto de la desposesión. 
Acumulación por desposesión

\begin{tabular}{|c|c|c|c|c|}
\hline \multirow{2}{*}{\multicolumn{2}{|c|}{ 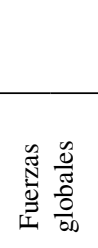 }} & $\begin{array}{l}\text { Por contaminación } \\
\text { (Río Lingue) }\end{array}$ & $\begin{array}{l}\text { Por división territorial } \\
\text { (Río Chaihuín) }\end{array}$ & $\begin{array}{l}\text { Por regulación } \\
\text { (Río Llesquehue) }\end{array}$ \\
\hline & & $\begin{array}{l}\text { Celulosa (la industria de mayor } \\
\text { crecimiento a nivel mundial). }\end{array}$ & $\begin{array}{l}\text { Forestal (industria global que } \\
\text { determina el uso del suelo y los } \\
\text { recursos naturales donde opera). }\end{array}$ & $\begin{array}{l}\text { Recursos del mar (la industria más } \\
\text { globalizada). }\end{array}$ \\
\hline \multicolumn{2}{|c|}{ 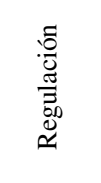 } & $\begin{array}{l}\text { Ley Base del Medio Ambiente } \\
\text { (1994) establece mecanismos para } \\
\text { evaluar impactos ambientales. } \\
\text { Código de Aguas (1981): } \\
\text { Concesiona el agua. }\end{array}$ & $\begin{array}{l}\text { Decreto } 701(1979) \text {, subsidia la } \\
\text { plantación de especies exóticas. }\end{array}$ & $\begin{array}{l}\text { Ley de Pesca y Acuicultura (1989): } \\
\text { regula los cultivos y la explotación } \\
\text { de los recursos del mar. }\end{array}$ \\
\hline \multicolumn{2}{|c|}{ 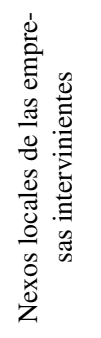 } & $\begin{array}{l}\text { No hay - Creados a través } \\
\text { del dinero y de "relaciones } \\
\text { permanentes y de mutua } \\
\text { colaboración... donde se } \\
\text { encuentran nuestras operaciones, } \\
\text { incorporando sus inquietudes } \\
\text { y necesidades en nuestra toma } \\
\text { de decisiones y apoyando su } \\
\text { desarrollo" (ARAUCO 2008). }\end{array}$ & $\begin{array}{l}\text { Alianzas con grupos locales vía } \\
\text { asignación de recursos. }\end{array}$ & $\begin{array}{l}\text { Formación de sindicatos } \\
\text { de orientación empresarial } \\
\text { que proveen a las empresas } \\
\text { exportadoras de productos del mar. }\end{array}$ \\
\hline \multicolumn{2}{|c|}{ 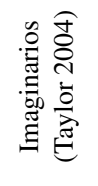 } & $\begin{array}{l}\text { La intervención se justifica como } \\
\text { progreso y se enfatiza la pobreza } \\
\text { local como un problema a resolver. }\end{array}$ & $\begin{array}{l}\text { Uso racional de los recursos } \\
\text { naturales: población al servicio de } \\
\text { la explotación. }\end{array}$ & $\begin{array}{l}\text { Sustentabilidad, } \\
\text { empresarialización. }\end{array}$ \\
\hline \multicolumn{2}{|c|}{ 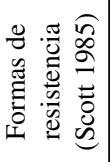 } & $\begin{array}{l}\text { Comité de Defensa del Mar como } \\
\text { expresión de soberanía. }\end{array}$ & $\begin{array}{l}\text { Por omisión. La comunidad se } \\
\text { abstiene de participar. }\end{array}$ & $\begin{array}{l}\text { Por transgresión, robo de material } \\
\text { (matuteo). }\end{array}$ \\
\hline \multirow{3}{*}{ 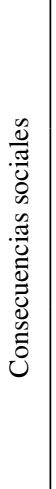 } & 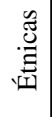 & $\begin{array}{l}\text { Enfrentamiento entre vecinos } \\
\text { y conflictos con una fuerte } \\
\text { connotación étnica. }\end{array}$ & $\begin{array}{l}\text { Conflictos entre grupos familiares } \\
\text { con componentes étnicos. }\end{array}$ & $\begin{array}{l}\text { Conflictos étnicos y por grupos de } \\
\text { actividad económica. }\end{array}$ \\
\hline & 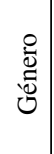 & $\begin{array}{l}\text { Mujer aparece como reserva moral } \\
\text { y defensora final de la comunidad. }\end{array}$ & $\begin{array}{l}\text { Se limita el campo laboral de la } \\
\text { mujer al ámbito de la vivienda. } \\
\text { Negación de su rol productivo. }\end{array}$ & $\begin{array}{l}\text { Exclusión de la mujer, restricción } \\
\text { de su área de trabajo, trabajo } \\
\text { subordinado a las actividades de } \\
\text { la pesca. }\end{array}$ \\
\hline & 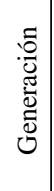 & $\begin{array}{l}\text { Se manipula a los antiguos, } \\
\text { forzando la radicalización } \\
\text { de los jóvenes }\end{array}$ & $\begin{array}{l}\text { Se privilegia a los jóvenes, } \\
\text { transfiriéndoles poder y } \\
\text { marginalizando a los antiguos. }\end{array}$ & $\begin{array}{l}\text { Se privilegia a los jóvenes } \\
\text { educados, transfiriéndoles poder. } \\
\text { Los antiguos y la población no } \\
\text { educada queda marginada del } \\
\text { proceso. }\end{array}$ \\
\hline 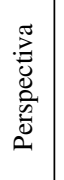 & & $\begin{array}{l}\text { Expulsión, inviabilidad, } \\
\text { contaminación. Perjudicados } \\
\text { directos son quienes se } \\
\text { especializan en la extracción } \\
\text { de recursos del mar. }\end{array}$ & $\begin{array}{l}\text { Nuevas dinámicas de ocupación, } \\
\text { conflictos latentes, explotaciones } \\
\text { heterogéneas con limitadas } \\
\text { posibilidades. }\end{array}$ & $\begin{array}{l}\text { Emergencia de respuestas } \\
\text { orgánicas, propuestas desde la } \\
\text { población, conflictos latentes, } \\
\text { sobreexplotación del recurso. }\end{array}$ \\
\hline
\end{tabular}


Todas las intervenciones expresan la operación de intereses globales: la pulpa de papel, la madera y la pesca son protagónicos en la economía mundial. Estas intervenciones están amparadas por la legalidad vigente, lo que posiciona al Estado en una situación contraria a los intereses de la comunidad. Estas intervenciones tienen una justificación ideológica que proyecta en la opinión pública una imagen favorable a su desarrollo y naturalizan el capitalismo como forma de vida, difuminando la experiencia histórica precapitalista, la que en adelante será denostada (Eiss 2008). La metáfora del progreso es la más poderosa cuando se justifica la desposesión por contaminación. Se describe a la población a ser intervenida como pobre y el desarrollo generado por la gran industria está llamado a modernizarla, siendo la educación, la infraestructura y el deporte las áreas privilegiadas para ello. En el ordenamiento territorial opera la racionalidad como expresión del pensamiento moderno aplicado al manejo de los recursos. La población se "ordena" (Scott 1998), a fin de asegurar los recursos para el mercado. El empresarialismo, a su vez, es la ideología que promueve el Estado: el sindicato más que político es empresario y su función es la de administrar las áreas de manejo. Estas representaciones ideológicas tienen un sesgo patriarcal quedando la mujer excluida de los posibles beneficios: es el último resorte moral de la comunidad amenazada (Lingue); o restringida a labores de repostería (Huiro), o excluida de las principales fuentes de ingresos relacionados a la pesca (Pucatrihue). La mujer sólo asume la dirección del sindicato cuando éste es débil o constituye fuerza unitaria cuando la división o el enredo se han hecho parte de la vida comunitaria.

Las conexiones locales son necesarias y las más destructivas se basan en el uso del dinero para obtener obsecuencia. Desde un punto de vista paisajístico, la situación presagia la creación de un gran desierto industrial en las áreas estuariales, como sería el caso de la evacuación de los desechos de CELCO en el mar. Distinta es la creación de alianzas locales a través de los planes de manejo y división territorial. En estas situaciones algún segmento comunitario adquiere protagonismo, haciéndose cargo de la propuesta y, eventualmente, tornarla a su favor. Cuando la desposesión se produce por regulación una fracción de la población adquiere protagonismo de carácter autonómico. Este espacio abre la posibilidad para generar, en un contexto de conflicto, espacios de negociación.
Los impactos sociales generados son preocupantes. El enredo se torna condición de operación de los sistemas locales, los que, a decir de la Real Academia de la Lengua (2007): "Dificultosamente se pueden desenredar y poner corrientes". Al enredo sucede la guerra interna, y el repliegue se produce hacia el mundo indígena, donde las y los pobladores sortean los escollos mediante la explotación de recursos diversos.

¿De qué modo influye el paisaje local en las posibilidades de los actores locales? El paisaje es ambiente y memoria, es recurso y espacio de vida. Las poblaciones tratan de recuperar lo que se les quita resignificando el paisaje y reorientando sus actividades, lo que en un ambiente estuarial es posible desarrollar. La resistencia a la expansión capitalista se apoya en formas hetárquicas de producción: mientras el enclave civilizatorio, con predominio chileno, se constituye monoproductivamente, los bolsones de resistencia se sostienen en la combinación de la leñería, la agricultura y ganadería, la acuicultura, la recolección de orilla, el turismo, los subsidios estatales y los proyectos de desarrollo. Los estuarios presentan, pues, una dualidad paisajística entre un enclave civilizador y una población dispersa de vocación rural. El dinamismo del enclave, con intensos períodos de cambio, migración y repoblamiento, contrasta con la resiliencia de la población dispersa que es capaz de reconstituirse en escenarios inestables. En los tres casos, el enclave civilizador se sitúa en la ribera que favorece la actividad pesquera, el principal elemento dinamizador de estos territorios, actividad que en la profundidad histórica se yuxtapone con la de los antiguos balnearios, hoteles y casas de veraneo.

\section{Conclusiones}

La acción de la empresa y del Estado en ambientes de confluencia histórica y ecológica procura desarticular las redes locales; sin embargo, la plurifuncionalidad de los paisajes estuariales posibilita la resiliencia de poblaciones que, de lo contrario, se verían desplazadas de sus territorios. Tales paisajes son amenazados por la expansión capitalista, la cual se asocia a una jerarquización constituida en torno al eje local dinamizador, estimulado por el asistencialismo, la cooptación o por la formación de núcleos empresariales, modalidades vinculadas a distintas prácticas de desposesión: por contaminación, por ordenamiento territorial y por regulación. $\mathrm{La}$ 
expansión capitalista resiente las relaciones locales; en algunos casos lo hace de modo irreversible y en otros permite la emergencia de actores que pueden torcer el curso de los acontecimientos.

El desmembramiento producido por la intrusión capitalista socava las bases ecológicas (merma de recursos) y sociales (hostilidad al interior de la comunidad) de sustentabilidad. El impacto de la desposesión es desigual: la acumulación por contaminación es devastadora, la ejercida a través del ordenamiento territorial y de la regulación permiten conservar en parte recursos estratégicos para la población. Frente a los procesos de acumulación por desposesión, la configuración del paisaje permite a las poblaciones maniobrar con autonomía relativa. Tales procesos son multiformes e impactan diferencialmente a las poblaciones locales y su medio ambiente.

El enredo es el medio preferente de desposesión en áreas que se tornan atractivas para el mercado y su instigación provoca conductas hostiles al interior de la comunidad. Se desmantela la armazón social asociada históricamente a un uso sustentable de los recursos. Para proteger los estuarios y sus poblaciones es preciso, desde la perspectiva de las iniciativas públicas y privadas, fortalecer las asociaciones paisajísticas fundadas en una organización hetárquica. Se requiere que las instituciones del desarrollo, la conservación y del gobierno comunal privilegien iniciativas que a ello propendan.

Agradecimientos: Los resultados son producto del proyecto FONDECYT F-1060111: "Paisajes Estuariales: Estrategias Adaptativas de las Poblaciones Locales y el Modelado Social de los Ecotonos Costeros de la X Región Norte y su Transformación a partir de la Legislación Pesquera". Agradecemos al Centro Transdisciplinario de Estudios Ambientales y Desarrollo Humano Sostenible de la UACh por el apoyo para la realización de esta investigación; a Marisela Pilquimán y Daniela Pino por su colaboración en la recolección de la información de campo, y a Iris Avillanosa por la confección del mapa. Asimismo, a quienes generosamente revisaron este manuscrito. Quisiéramos, finalmente testimoniar nuestro reconocimiento a las comunidades que hoy padecen las consecuencias de procesos sobre los que ellas carecen de control, así como a los evaluadores y/o evaluadoras que generosamente brindaron su tiempo para revisar este artículo.

\section{Referencias Citadas}

Aasbŏ, S.

1999 History and ecology in everyday landscape. Norwegian Journal of Geography 53:145-152.

Accatino, D.

2009 Regímenes legales de acceso y explotación de recursos hidrobiológicos, hídricos y mineros en los estuarios de los ríos Queule, Lingue, Chaihuín y Llesquehue. Manuscrito en posesión del autor.

Adrienne, R.

2008 Privatizing social reproduction: The primitive accumulation of water in an era of neoliberalism. Antipode 40:535-560. ARAUCO

2008 Código de Ética. Aprobado por el Directorio de Celulosa Arauco y Constitución S.A. http://www.arauco.cl/_file/ file_1585_etica_esp.pdf (14 de abril de 2009).

Balée, W.

1998 Historical ecology: Premises and postulates. En Advances in Historical Ecology, editado por W. Balée, pp. 13-29. Columbia University Press, New York.

Bengoa, J.

1996 Historia del Pueblo Mapuche (siglo XIX y XX). Ediciones Sur, Santiago.

Chihuailaf, E.

1999 Recado Confidencial a los Chilenos. LOM. Santiago.
Concha, M.

1998 Una mirada a la identidad de los grupos Huilliche de San Juan de la Costa. Documentos de Trabajo. Universidad Arcis, Santiago.

Criado, $\mathrm{F}$.

1999 Del Terreno al Espacio: Planteamientos y Perspectivas para la Arqueología del Paisaje. Grupo de Investigación en Arqueología del Paisaje. Universidad de Santiago de Compostela, Santiago de Compostela.

Crumley, C.L.

1994a The ecology conquest: Contrasting agropastoral and agricultural societies. Adaptation to climatic change. En Historical Ecology: Cultural Knowledge and Changing Landscapes, editado por C.L. Crumley, pp. 183-201. School of American Research Press, Santa Fe, New Mexico.

1994b Historical ecology: A multidimensional ecological orientation. En Historical Ecology: Cultural Knowledge and Changing Landscapes, editado por C.L. Crumley, pp. 1-16. School of American Research Press, Santa Fe, New Mexico.

Descola, Ph.

1996 La Selva Culta. Simbolismo y Praxis en la Ecología de los Achuar. Traducido por J. Carrera, X. Catta Quelen, y F. Illouz. Abya-Yala, Quito. 
Duhart, P., M. McDonough, J. Muñoz, M. Martin y M. Villenueve 2001 El complejo metamórfico Bahía Mansa en la Cordillera de la Costa del centro-sur de Chile $\left(39^{\circ} 30^{\prime}-42^{\circ} 00^{\prime} \mathrm{S}\right)$; geocronología K-Ar, 40Ar/ 39Ar y U-Pb e implicancias en la evolución del margen sur-occidental de Gondwana. Revista Geológica de Chile 28:179-208.

Eiss, P.K.

2008 Beyond the object. Of rabbits, rutabagas and history. Anthropological Theory 8:79-97.

Escobar, A.

1999 'After nature': Steps to an anti-essentialist political ecology. Current Anthropology 40:1-30.

Fine, B.

2006 Debating the 'new' imperialism. Historical Materialism 14:133-156

Fischer-Kowalski, M.

2002 El metabolismo de la sociedad: sobre la infancia y adolescencia de una naciente estrella conceptual. En Sociología del Medio Ambiente, editado por M. Redclift and G. Woodgate, pp. 119-141. McGraw-Hill, Madrid.

Foerster, R.

1985 Vida Religiosa de los Huilliches de San Juan de la Costa. Rehue, Santiago.

2004 ¿Pactos de Sumisión o Actos de Rebelión? Una Aproximación Histórica y Antropológica a los Mapuches de la Costa de Arauco, Chile. Tesis Doctoral, Universiteit Leiden, Leiden.

Godoy, $\mathrm{M}$.

2003 Informe diagnóstico social localidades costeras provincia de Valdivia. World Wildlife Fund, Programa Ecorregión de los Bosques Templados Valdivianos, Valdivia.

Gudeman, S.

2008 Economy's Tension. The Dialectics of Community and Market. Berghahn Books, New York, Oxford.

Harvey, D.

2003 The New Imperialism. Oxford University Press, Oxford, New York.

2004 El "nuevo" imperialismo. Sobre reajustes espacio temporales y acumulación mediante desposesión. Revista Herramienta (27). http://www.herramienta.com.ar/modules. php $?$ op $=$ modload $\&$ name $=$ News $\&$ file $=$ article $\&$ sid $=301$, (6 de octubre de 2007).

2005 Acumulación mediante desposesión. Revista Herramienta (29). http://www.herramienta.com.ar/modules.php?op=mo dload $\&$ name $=$ News $\&$ file $=$ article $\&$ sid $=286,(6$ de octubre de 2007).

2006 Neo-liberalism as creative destruction. Geografiska Annaler Series B: Human Geography 88:145-158.

Hirsch, E. y M. O'Hanlon

1995 The Anthropology of Landscape: Perspectives on Space and Place. Clarendon Press, Oxford.

IFOP

2009 Áreas de manejo.http://www.ifop.cl/areas_manejo.html (15 de abril de 2009).

Instituto Nacional de Estadísticas (INE)

2006 Censo de Población y Vivienda, 2002, CELADE-División de Población, CEPAL Instituto Nacional de Estadística, Santiago.

Ingold, $\mathrm{T}$.

1995 Building, dwelling, living. How animals and people make themselves at home in the world. En Shifting Contexts.
Transformations in Anthropological Knowledge, editado por M. Strathern, pp. 57-80. Routledge, London and New York.

Klein, J.A. y S.A. Wolf

2007 Toward multifunctional landscapes: cross-sectional analysis of management priorities in New York's northern forest. Rural Sociology 72:391-417.

Lafkenche, Equipo Técnico Identidad Territorial

2005 Proyecto de ley que crea el espacio marítimo de pueblos originarios. Manuscrito en posesión de los autores.

Lara, A., D. Soto, J. Armesto, P. Donoso, P. Wermli, L. Nahuelhual y F. Squeo

2003 Componentes Científicos Clave para una Política Nacional sobre Usos, Servicios y Conservación de los Bosques Nativos Chilenos. Universidad Austral de Chile, Valdivia.

Le-Breton, Y. y M.C. Flores

2007 Historia social de la localidad de Huiro. Manuscrito en posesión de los autores.

Lipietz, A.

2002 ¿Qué es la Ecología Política? La Gran Transformación del S.XXI. LOM e IEP, Santiago.

Little, P.E.

1999 Environments and environmentalists in anthropological research: Facing a new millennium. Annual Review in Anthropology 28:253-284.

Malinowski, B.

1966 [1922] Argonauts of the Western Pacific. Routledge \& Kegan Paul, London.

Marx, K.

1980 [1867] El Capital: Crítica de la Economía Política. Traducido por Scaron, P., Siglo XXI, Madrid.

McGovern, T.H

1994 Management for extinction in Norse Greenland. En Historical Ecology: Cultural Knowledge and Changing Landscapes, editado por Crumley, C.L. pp. 127-154. Society of American Research Press, Santa Fe, New Mexico.

\section{MIDEPLAN}

2003 Las trayectorias del desarrollo humano en las comunas de Chile. En Temas de Desarrollo Humano Sustentable. Ministerio de Planificación Nacional, Santiago.

Molina, R., M. Correa, C. Smith-Ramírez y A. Gainza

2006 Alerceros Huilliche de la Cordillera de la Costa. Andros Impresores, Santiago.

Moreno, C. y A. Fedele

2003 Ecosistemas marinos y del borde costero. En Estado del Medio Ambiente en Chile 2002, editado por el Centro de Análisis de Políticas Públicas, Universidad de Chile, pp. 251-295. Lom, Santiago.

Patterson, T.C.

1994 Toward a properly historical ecology. En Historical Ecology: Cultural Knowledge and Changing Landscapes, editado por Crumley, C.L., pp. 223-237. Society of American Research Press, Santa Fe, New Mexico.

Perillo, G.

1995 Definitions and geomorphologic classification of estuaries. En Geomorphology and Sedimentology of Estuaries., editado por G. Perillo, pp. 14-47. Developments in Sedimentology. Elsevier, Amsterdam.

Polanyi, K.

1944 The Great Transformation. Farrar \& Rinehart, New York, Toronto. 
Quiroz, D. y J.C. Olivares

1987 Amuatan Pucatra Agüelito Huentiao, Amuatan Pucatra. Permanencia de una pauta adaptativa en San Juan de la Costa. Boletín del Museo Mapuche de Cañete 3:13-26.

Real Academia Española de la Lengua

2007 [1732] Diccionario Autoridades. http://buscon.rae.es/ ntlle/SrvltGUIMenuNtlle? $\mathrm{cmd}=$ Lema\&sec=1.0.0.0.0., (3 de diciembre de 2007).

Ridgeway, J.

2004 It's All for Sale. The Control of Global Resources. Duke University Press, Durham and London.

Sauer, C.

1925 The morphology of landscape. University of California Publications in Geography 2:19-54.

Scott, J.C.

1985 Weapons of the Weak: Everyday Forms of Peasant Resistance. Yale University Press, New Haven.

1998 Seeing Like a State: How Certain Schemes to Improve the Human Condition Have Failed. Yale University Press, New Haven.

Sellin, E.

1996 A congruence of landscape and the mind. Literary Review 39:492-503.

Short, A.D.

1979 Three dimensional beach-stage model. Journal of Geology 87:553-571.

Skewes, J.C.

2001 Reconversiones de otro tiempo: la vernacularización de los Altos Hornos de Corral. Proposiciones 32:302-321.

Skewes, J.C. y D.E. Guerra

2004 The defense of Maiquillahue bay: Knowledge, faith, and identity in an environmental conflict. Ethnology 43:217-332.

Skewes, J.C. y M. Silva

2007 Elementos para una comprensión ecológico-cultural de las narraciones míticas acerca de las aguas y los seres del agua en la cosmovisión mapuche huilliche. En Sociedad y Cultura: Reflexiones Transdisciplinarias, editado por C. Rodríguez, R. Browne, C. del Valle y S. Figueroa, pp. 129-138. UACh, UFRO, MECESUP, Valdivia.

Sparke, M.

2008 Political geography-political geographies of globalization III: Resistance. Progress in Human Geography 32:423440.
Stokowski, P.

2002 Languages of place and discourses of power: Constructing new senses of place. Journal of Leisure Research 34:36883.

Subiabre, A. y C. Rojas 1994 Geografía Física de la Región de Los Lagos. Universidad Austral de Chile, Valdivia.

Taussig, M.T.

1980 The Devil and Commodity Fetishism in South America. University of North Carolina Press, Chapel Hill.

Taylor, Ch.

2004 Modern Social Imaginaries. Duke University Press, London.

Tecklin, D. y A. Farías

2003 Caracterización preliminar de los predios ChaihuínVenecia, Cordillera de la Costa, Décima Región. WWF, Valdivia.

Thornton, Th.F.

1997 Know your place: The organization of Tlingit geographic knowledge. Ethnology 36:295-308.

Tuan, Y.F.

1977 Space and Place. University of Minnesota Press, Minneapolis.

Turner, $\mathrm{T}$.

2008 Marxian value theory: An anthropological perspective. Anthropological Theory 8:43-56.

Vasey, D.E.

1998 Uncommon ground: cultural landscapes and environmental values uncommon ground: cultural landscapes and environmental values. American Anthropologist 100:10711072.

Vergara, J.I., A. Mascareño y R. Foerster 1996 La Propiedad Huilliche en la Provincia de Valdivia. Corporación Nacional de Desarrollo Indígena, Santiago de Chile.

Zedeno, M., D. Austin y R. Stoffle

1997 Landmark and landscape: A contextual approach to the management of American Indian resources. Culture \& Agriculture 19:123-129.

Zsuzsa, G. y S. Ó Riain

2002 Global ethnography. Annual Review of Sociology 28:271-95.

\section{Nota}

1 Recordemos que el Diccionario de Autoridades de 1732, de la Academia Española de la Lengua, define en su página 485 el verbo enredar como: "Por metáfora vale descomponer, meter cizaña y discordia entre unos y otros para revolverlos entre sí: lo que asimismo se dice de los negocios y dependencias cuando las confunden y revuelven de calidad que dificultosamente se pueden desenredar y poner corrientes" (RAE A 1732: 485, 1. http://buscon.rae. es/ntlle/SrvltGUIMenuNtlle?cmd=Lema\&sec=1.0.0.0.0., [3 de diciembre de 2007]). 
